\title{
Carrier status of Acinetobacter among healthcare personnel and prevalence of the same in the environment
}

\author{
Gedam DS ${ }^{1}$, Patel $\mathbf{U}^{2}$ \\ ${ }^{1}$ Dr. D Sharad Gedam, Professor, RKDF Medical College, Bhopal, ${ }^{2}$ Dr Umesh Patel, Professor, RKDF Medical College, \\ Bhopal, MP, India
}

Address for Correspondence: ${ }^{1}$ Dr. Sharad Gedam, Email: sharad.gedam@gmail.com

\begin{abstract}
Background: Acinetobacter baumanii is an increasingly troublesome nosocomial pathogen, often with multidrug and pandrug resistant strains, mainly in intensive care units (ICUs), being responsible for different types of nosocomial infections. Objectives: The objective was to determine prevalence of carriers of Acinetobacter Spp. and predisposing risk factors among healthcare workers (HCWs) in ICUs and their role as source in nosocomial infection. Materials and Methods: Specimens from nose, throat, axilla and hands of all HCWs (55 (40 staff nurses, 15 attenders)) from ICUs and $30 \mathrm{HCWs}$ from general wards were collected and processed by standard laboratory procedures. Results: Prevalance of A.baumanii carriers was $18.18 \%$. Higher carrier rate was observed in male than female HCWs $(12.72 \%$ Vs $5.5 \%)$. carrier rate was $22.2 \%$ and $18.75 \%$ in SICU and PICU respectively with none from general wards. Carrier rate was $7.3 \%$ at axilla and hands.Conclusion:Health care workers are responsible for colonization of hands by various hospital organism followed by spread to other contacts.
\end{abstract}

Key words: Acinetobacter, Carriers, Healthcare workers, ICUs, Nosocomial infections.

\section{Introduction}

Acinetobacter baumanii (A.baumanii) is an increasingly troublesome nosocomial pathogen, often with multidrug and pan-drug resistant strains, mainly in intensive care units (ICUs) being responsible for different types of nosocomial infections with increasingly limited therapeutic options, higher mortality and morbidity [1] Incidence of acinobacter has increased dramatically in last 2 decades specially in ICU settings. Their favourable growth environment in ICU further increases concern over this issue [2]. There are very few studies available on acinobacter despite of global concern.

Being an important organism related with hospital acquired infections specially in ICU setting it is important that health care staff should be fully aware about this [2]. It is largely identified from the hospital stall, environment \& equipment used in hospital [2]. They are known for hospital outbreaks earlier also. Health care staff are solely responsible for spread of this resistant organism isolated from ICU settings mainly. Spread by hands \& skin from hospital staff is

Manuscript received: $6^{\text {th }}$ July 2017

Reviewed: $16^{\text {th }}$ July 2017

Author Corrected; $24^{\text {th }}$ July 2017

Accepted for Publication: $28^{\text {th }}$ July 2017 widely prevalent. The significant environmental reservoirs in the ICU include walls, ventilators, resuscitation bags, mattresses, hand-washing sinks, gowns and gloves [2].

\section{Materials and Methods}

Study Design and Setting- This prospective observational study was carried out from July 2015 to December 2015, in the tertiary care teaching hospital.

Study Population, Sample Size, and Sampling Strategy- The study population consisted of HCWs working in different ICUs of the hospital. A total of 220 random specimens were collected from $55 \mathrm{HCWs}$, consisting of 40 staff nurses, 15 attenders/ayyas from ICCU, NICU, surgical ICU (SICU). 120 specimens from $30 \mathrm{HCWs}$ from general wards were also collected and processed to determine the carrier state.

The presentcarrier study among HCWs was conducted in ICUs of our hospital with the patients suffering from nosocomial infections namely; catheter associated urinary tract infection, ventilator associatedpneumonia, 


\section{Original Research Article}

lower respiratory tract infection (LRTI), chronic obstructive pulmonary disease with LRTI. Study was conducted in our ICUs witnessing higher incidence of culture proven nosocomial infections due to Acinetobacter species.

Specimen Collection- Four specimens per HCW were collected, namely web spaces of hands, axilla, throat, and nose by using a sterile swab soaked in sterile normal saline as per standard laboratory procedures [3].

Institutional ethical committee clearance was obtained for conducting the study. Informed consentwas obtained from HCWs before sample collection.

HCWs with $<6$ months experience in our hospital and HCWs suffering from an infectious disease were excluded from the study.

HCWs with isolation of Acinetobacter spp. from at least one of the body sites namely, nose, throat, axilla, and hands were considered as carriers for Acinetobacter spp.

Hospital samples were collected from ventilatory circuit, suction apparatus, beds, floor, air-conditioner vents, medicine/dressing trolleys, window stills, door handles, wash basins. Four swabs were collected from each site.

Microbiological Processing- Samples from health care workers were collected with pre moistened swabs. Environmental samples from dry surfaces were taken with absorbent cotton-wool swabs, which were moistened with peptone water. The sample swabs were inoculated in enriched brain heart infusion broth and incubated for 24 hours at $37^{\circ} \mathrm{C}$.

After incubation the broth was subculture on 5\% sheep blood agar and MacConkey agar. The bacteria isolated from all these cultures were identified based on standard bacteriological techniques.

The susceptibility of Acinetobacter spp. isolates was performed on Muller-hinton agar by Kirby-bauer disc diffusion method as per clinical laboratory standards institute guidelines [4].

Antibiotics used were AMC (Amoxycillin+clavalunic acid), GEN(Gentamycin), CIP (Ciprofloxacin), CTX (Cefotaxime), CXM (Cefuroxime), NT (Netilmycin), CAZ(Ceftazidime), PTZ (Piparacillin + Tazobactum), CIS(Ceftrioxone).

Typing of Isolates From Carriers-Typing of Acinetobacter spp. was done by antibiogram typing. Association of Acinetobacter spp. carrier with different nosocomial infections in different areas of the hospital was done by identical antibiogram type of Acinetobacter spp. from carriers and patients.

Results were quantitated by analysing number of cases of nosocomial infections associated with antibiogram type of Acinetobacter spp. fromcarriers by retrospective review of case sheets and prospective observation.

\section{Results}

The present study reveals a prevalence of $18.18 \%(10 / 55)$ for A. baumanii carriers among HCWs (nurses and attenders/ayyas), working in different ICUs of our tertiary care hospital. None of the carriers for other Acinetobacter spp. were observed. Higher carrier rate was observed among male than female $\operatorname{HCWs}(8 / 55)$ vs $(2 / 55)$, and higher rate in nurses than attenders $(7 / 55)$ Vs $(3 / 55)$.

Distribution of carrier rates in different ICUs was not statistically significant. Carrier rate of A. baumanii was highest in SICU, 22.2\%(2/9) followed by ICCU, 20\%(1/5) and MICU, 20\% (3/15) and was least inNICU, 10\%(1/10).

Carrier rate ataxilla and hands was 7.27\% (4/55) followed by throat 5.5\% (3/55), nose 1.8\% (1/55). Three antibiogram types of A. baumanii were observed and AB-1 (6 strains) were resistant to all drugs.

These strains were associated with majority of nosocomial infections. AB-2 (3 strains) were sensitive to meropenem, resistant to others, AB-3 (1 strain) was sensitive to all antibiotics.

Antibiotics used were AMC (Amoxycillin+clavalunic acid), GEN (Gentamycin), CIP (Ciprofloxacin), CTX (Cefotaxime), CXM (Cefuroxime), NT (Netilmycin), CAZ (Ceftazidime), PTZ (Piparacillin+ Tazobactum), CIS (Ceftrioxone). 
Original Research Article

Table-1: Distribution of Acinetobacter carriers in ICUs

\begin{tabular}{|c|c|c|}
\hline Name of ICU & Total number of HCWs studied & Acinetobacter carrier rate (\%) \\
\hline ICCU & 05 & $1(20)$ \\
\hline MICU & 15 & $3(20)$ \\
\hline NICU & 10 & $1(10)$ \\
\hline SICU & 09 & $2(22.2)$ \\
\hline PICU & 16 & $3(18.8)$ \\
\hline Total & $\mathbf{5 5}$ & $\mathbf{1 0}(\mathbf{1 8 . 1 8})$ \\
\hline
\end{tabular}

Table-2: Distribution of carriers at different body sites

\begin{tabular}{|c|c|}
\hline Body sites & Acinetobacter carrier rate (\%) \\
\hline Nose & $1(1.8)$ \\
\hline Throat & $3(5.5)$ \\
\hline Axilla & $4(7.27)$ \\
\hline Hands & $4(7.27)$ \\
\hline
\end{tabular}

One strain of A.baumanii was isolated from ventilatory circuit, suction apparatus, wash basin each and two strains were isolated from medicine/dressing trolley.

\section{Discussion}

Acinobacter is becoming more common resitant organism isolated from hospital. Little is known abut economic burden caused by this organism in comparison to othrs (eg, MRSA)[5].

Emerging infections due to Multidrug resistant strains of Acinetobacter spp. in the ICU is a growing therapeutic concern for clinicians worldwide. Inspite of several largescale multicentric studies, the source/ reservoirs, transmission dynamics and failure to control infections due to Acinetobacter spp. has remained an enigma in ICUs[1].

The present carrier study conducted in ICUs of tertiary care hospital highlights the role of apparently healthy HCWs as carriers of A. baumani, acting as source or reservoirs of this nosocomial pathogen. The present carrier study reports a prevalence of $18.8 \%$ (10/55) of carrier state.other studies have reported a variable carrier rates.

Presentstudy highlights the role of carrier of A.baumani at different body sites like, nose, axilla, hands and throat. Joseph et al, concluded that A.baumanii is known to survive in healthcare environment are very effective human colonizers[6]. Bayuga et al, have reported a very high prevalence of the carrier state for A. baumani $(26.66 \%)$ [10]. A. A. Hershan et al, reported a prevalence of carrier rate of $14.63 \%$ [1]. Few authors have been isolated Acinetobacter spp. in $27 \%$ of hospital sink taps and $20 \%$ of hospital floor swab cultures[6]. Marchaim Dhor et al, screened six body sites nostrils, pharynx, skin, rectum, wounds and endotrachial aspirates, reported sensitivity of $55 \%$, and sensitivities of single sites ranged from $13.5 \%-29 \%[7]$.

Lee et al, have reported a colonization prevalence of $0.5-4 \%[5]$. Joseph et al, have isolated A. baumanii from suction apparatus, floor, dressing trolley, door handle and VAP patients and have found that it can be a very effective human colonizer [8].

The quantitative antibiogram typing revealed that there were two clusters of Acinetobacter baumanii of which the MDR cluster is large, while very few isolates belonged to the succeptiblecluster[8].

A.baumanii has a higher propensity to be transmitted to HCWs than do other MDR bacteria. This may be a factor in nosocomial spread, explaining in part the recent worldwide emergence of MDR A. Baumanii[9].

It was observed that colonization of patients occur during admission in hospital specially in ICU setting. Later on it associated with spread of these organism by hand and skin contacts to other patients of health care staff [6].

The ICU environment and the equipment can get contaminated directly with the secretions/discharges from patients during various patient care activities or indirectly through the contaminated hands of $\mathrm{HCWs}[8]$. 


\section{Original Research Article}

Limitation of the Study- There are some limitations of this study. Most common is that we have not studied effect of hand hygine \& other control measures during study period; therefore, further studies are required to examine the various infection control measures and evaluate the usefulness of such measures. We could not elucidate the exact mode of transmission of Acinetobacter between the environment and the patients.

\section{Conclusion}

Acinobacter species are become more important organism responsible for hospital acquired infections. They adopt environment very rapidly and become resistant. It is expected that they will become more serious concern later on due to limited choice of antibiotics available. Awareness programes for health care staff and need for better hygine practices are core for prevention of HAI.

These practice including equipment decontamination, strict attention to hand washing and isolation procedures, and control of antibiotic usage, appears to be the combination of measures most likely to control the previously unabated spread of Acinetobacter spp. in hospitals.

\section{Funding: Nil, Conflict of interest: None. Permission of IRB: Yes}

\section{References}

1. Hershan Almonther A, Yogesh Babu KV, Jayanth SS, Manjula R. Role of carriers of Acinetobacter species in transmission of nosocomial infections in intensive care units. International $\mathrm{J}$ of Health system and Disaster Management 2014;2(4):210-215.

2. RungrunghiranyaS, Somboonwit C, Kanchanapoom T. Acinetobacter infection in the intensive care unit. $\mathrm{J}$ Infect Dis Antimicrob Agents 2005;22(2):77-92.
3. Fallon RJ, Young $\mathrm{H}$, Neisseria, Moraxella, Acinetobacter. In: Collee JG, editor. Mackie and Mc Cartney Practical Medical Microbioligy. 14th ed. Edinburg: Churchill Livingstone; 2006.p.294-6.

4. Clinical and Laboratory Standards Institute. Performance Standards for Antimicrobial Susceptibility Testing; Seventeenth Informational Suppliment. CLSI document M 100-S 17. Vol. 27. Suppl. 17. Wayne,Pa: Clinical and Laboratory Standards; 2007.

5. Lee BY, McGlone SM, Doi y, Bailey RR, Harrison LH. Economic value of Acinetobacter baumanii screening in the intensive care unit. Clinical Microbiology and Infection 2011;17(11):1691-1697.

6. Bergogne-Berezin E, Towner KJ. Acinetobacter spp. as nosocomial pathogens: microbiological, clinical, and epidemiological feauters. Clin. Microbiol. Rev 1996;9 (2): 148-165.

7. Marchaim D, Navon-Venezia S, Schwartz D, Tarabeia J, Fefer I, Schwaber MJ, Carmeli Y. Surveillance cultures and duration of carriage of multidrugresistant Acinetobacter baumannii. J Clin Microbiol. 2007 May;45(5):1551-5. Epub 2007 Feb 21.

8. Joseph NM, Sistla S, Dutta TK, Badhe AS, Rasitha D, Parija SC. Role of intensive care unit environment and health-careworkers in transmission of ventilatorassociated pneumonia. J Infect Dev Ctries. 2010 Jun 3;4 (5):282-91.

9. Morgan DJ, Liang SY, Smith CL, Johnson JK, Harris AD, Furuno JP, Thom KA, Snyder GM, Day HR, Perencevich EN. Frequentmulti drug-resistan Acineto-bacter baumanniicontamination of gloves, gowns, and hands of healthcare workers. Infect Control Hosp Epidemiol. 2010 Jul; 31 (7):716-21. doi: 10.1086/ 653201.

\section{How to cite this article?}

Gedam DS, Patel U.Carrier status of Acinetobacter among healthcare personnel and prevalence of the same in the environment. Trop J Ophthalmol Otolaryngol.2017;2(2):23-26. doi: 10.17511/jooo.2017.i02.02. 